\title{
Multi-axial load application and DIC measurement of advanced composite beam deformation behavior
}

\author{
V. Fedorov a and C. Berggreen
}

Department of Mechanical Engineering, Technical University of Denmark, Niels Koppels Alle, 2800 Kgs Lyngby, Denmark

\section{Abstract}

For the validation of a new beam element formulation, a wide set of experimental data consisting of deformation patterns obtained for a number of specially designed composite beam elements, have been obtained. The composite materials applied in the beams consist of glass-fiber reinforced plastic with specially designed layup configurations promoting advanced coupling behavior. Furthermore, the beams are designed with different cross-section shapes. The data obtained from the experiments are also used in order to improve the general understanding related to practical implementation of mechanisms of elastic couplings due to anisotropic properties of composite materials. The knowledge gained from these experiments is therefore essential in order to facilitate an implementation of passive control in future large wind turbine blades.

A test setup based on a four-column MTS servo-hydraulic testing machine with a maximum capacity of $100 \mathrm{kN}$ was developed, see Figure 1. The setup allows installing and testing beams of different cross-sections applying load cases such as axial extension, shear force bending, pure bending in two principal directions as well as pure torsion, see Figure 2. In order to apply multi-axial loading, a load application system consisting of three hydraulic actuators were mounted in two planes using multi-axial servo-hydraulic control. The actuator setup consists of the main actuator on the servo-hydraulic test machine working in the vertical axis (depicted on Figure 1) placed at the testing machine crosshead and used for application of vertical forces to the specimens. Two extra actuators are placed in a horizontal plane on the T-slot table of the test machine in different positions in order to apply loading at the tip of the specimen in various configurations.

In order to precisely characterize the global as well as surface deformations of the beam specimens tested, a combination of different measurement systems were used during the tests. Digital Image Correlation (DIC) systems [1] able to measure the 3D displacement field along the specimen surface were applied. Two linked DIC systems were used simultaneously during the experiments in order to obtain measurements for most of the surface of the beams. Additionally, an optical system based on mirrors and laser beams allowing direct measuring of the twist along the specimen were applied as well. Results from both systems were additionally used to verify obtained results.

A number of FE models with different modeling approaches were furthermore developed for each specimen type and validated against the experimental results. The models were also used to investigate inaccurate prediction of torsional behavior of composite structures when utilizing nodal offsets in shell element based models [2, 3].

${ }^{\mathrm{a}}$ E-mail : vlf@mek.dtu.dk

This is an Open Access article distributed under the terms of the Creative Commons Attribution-Noncommercial License 3.0, which permits unrestricted use, distribution, and reproduction in any noncommercial medium, provided the original work is properly cited. 
Finally, as a part of the post-processing, the DIC measurements were compared with FE results, using of a new software feature for evaluating DIC measurements against the FE results in the commercial DIC software ARAMIS. The direct comparison feature made it possible to investigate and compare differences between cross-sectional distortions in numerical and experimental results.
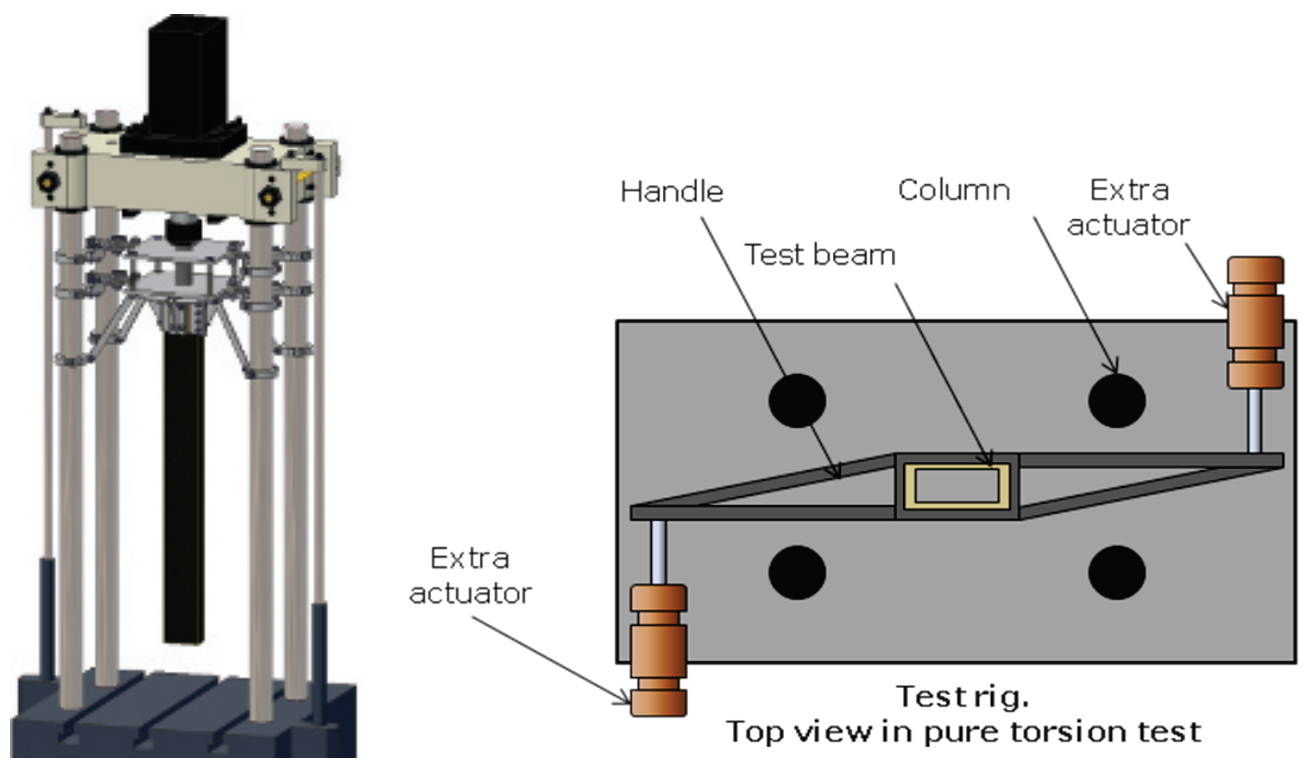

Fig. 1. Test setup based on four-column testing machine (left). Horizontal actuators and handle load introduction fixture shown for a pure torsion test setup (right).

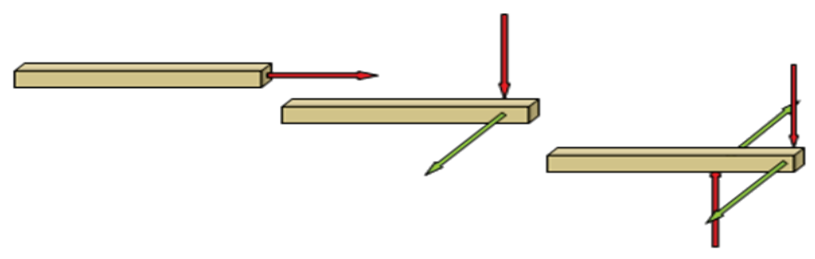

Fig. 2. Load cases, from left axial extension, multi-axial bending by shear forces, multi-axial pure bending and pure torsion.

\section{References}

1. M. A. Sutton, J-J. Orteu, H. Schreier, Image Correlation for Shape, Motion and Deformation Measurements, Springer (2009)

2. D. L. Laird, F. C. Montoya, D. J. Malcolm, Proceedings of AIAA/ASME Wind Energy Symposium, Finite Element Modeling of Wind Turbine Blades, AIAA-2005-0195, pp 9-17 (2005)

3. V. Fedorov, N. Dimitrov, C. Berggreen, S. Krenk, K. Branner, P. Berring, Proceedings of ICCM-17, Investigation of Structural Behavior due to Bend-Twist Couplings in Wind Turbine Blades (2009) 